\title{
Characteristics of newly diagnosed children with Type 1 diabetes - DKA versus Non- DKA presentation
}

\author{
Sarrah El Munshid ${ }^{1}$, Saji Alexander ${ }^{1}$, Karen Spowart ${ }^{1}$, Karen Logan², Kingi Aminu$^{1}$, Nicola Bridges ${ }^{1}$ \\ 'Department of Paediatric Diabetes and Endocrinology, Chelsea and Westminster Hospital NHS Foundation Trust \\ 2 Imperial College, London
}

\begin{abstract}
BACKGROUND
Diabetic Ketoacidosis (DKA) is a common presentation of newly diagnosed Type1 Diabetes

There is a world wide variation in the rate of DKA as first presentation of Type 1 diabetes of $16 \%$ to $67 \%$.

In the UK average frequency of DKA as first presentation is $25 \%$. DKA is a leading cause of mortality in children with type 1 diabetes
\end{abstract}

\section{AIMS / OBJECTIVES}

To compare the demographic and clinical characteristics of DKA versus non-DKA presentations in children $<16$ years at diagnosis of T1DM.

Compare newly diagnosed children with T1DM presenting with DKA versus Non - DKA to determine risk factors for DKA

\section{METHOD}

Retrospective audit covering a 30 month period; from January 2013 to June 2015 in a single paediatric diabetes unit.

\section{Results}

- Total number of new T1DM cases: 36

- DKA presentation :15 (42\%)

- Non DKA presentation:21 (58\%)

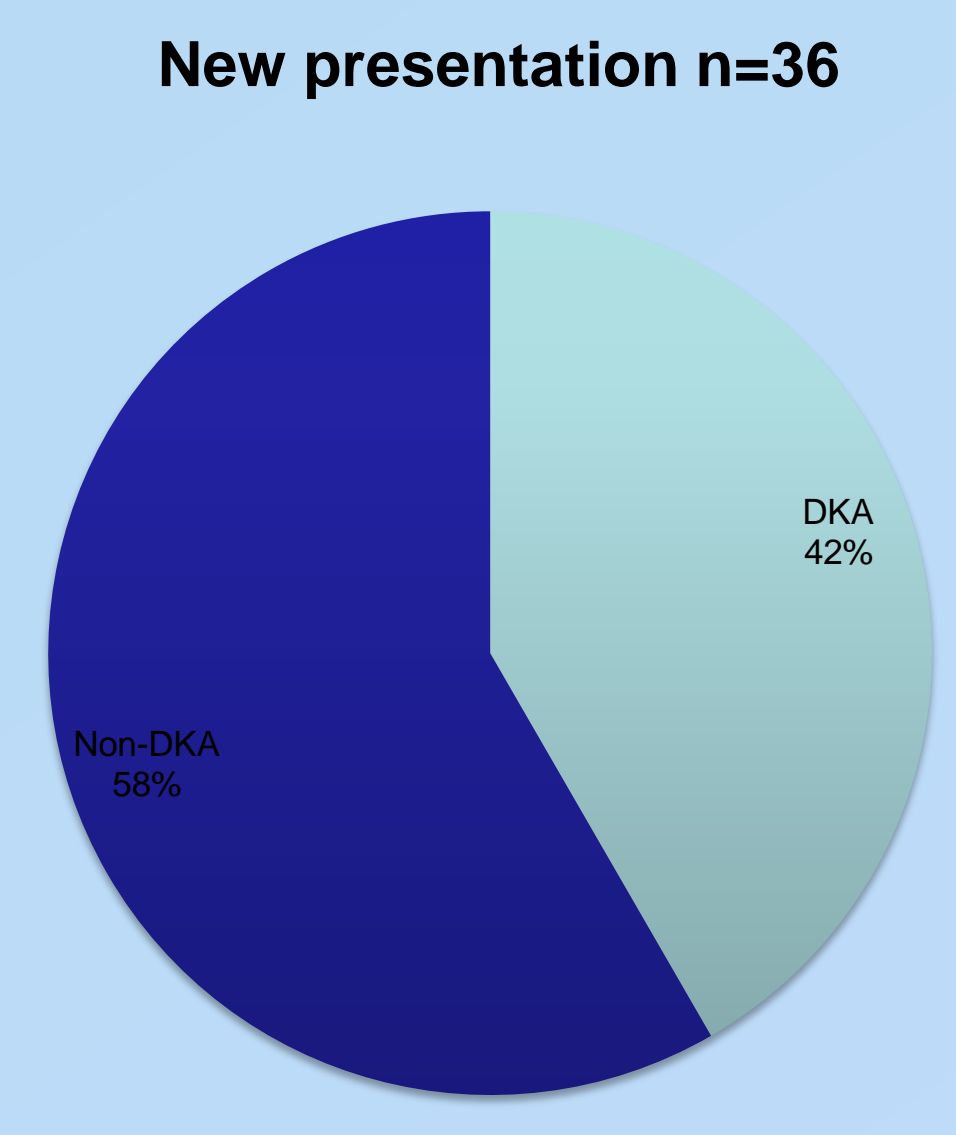

\section{Background data}

Sex

- Females 56\%; males $44 \%$

- Median age was 8 years

- $40 \%$ presented in mild DKA, $33 \%$ presented in moderate DKA, and $20 \%$ presented in severe DKA

- $40 \%$ of males presented in DKA versus $43 \%$ of females

Age

Peak age of diagnosis- 6 years

- $100 \%$ of children at 2 years presented in DKA

- There was a trend for younger children to present in DKA

- $66 \%$ of those aged $0-5$ years presented in DKA compared to $29 \%$ and $46 \%$ in $5-9$ years and $10-16$ years respectively

Age at presentation $(\mathrm{n}=36)$

Age of DKA+ pts (n=15)

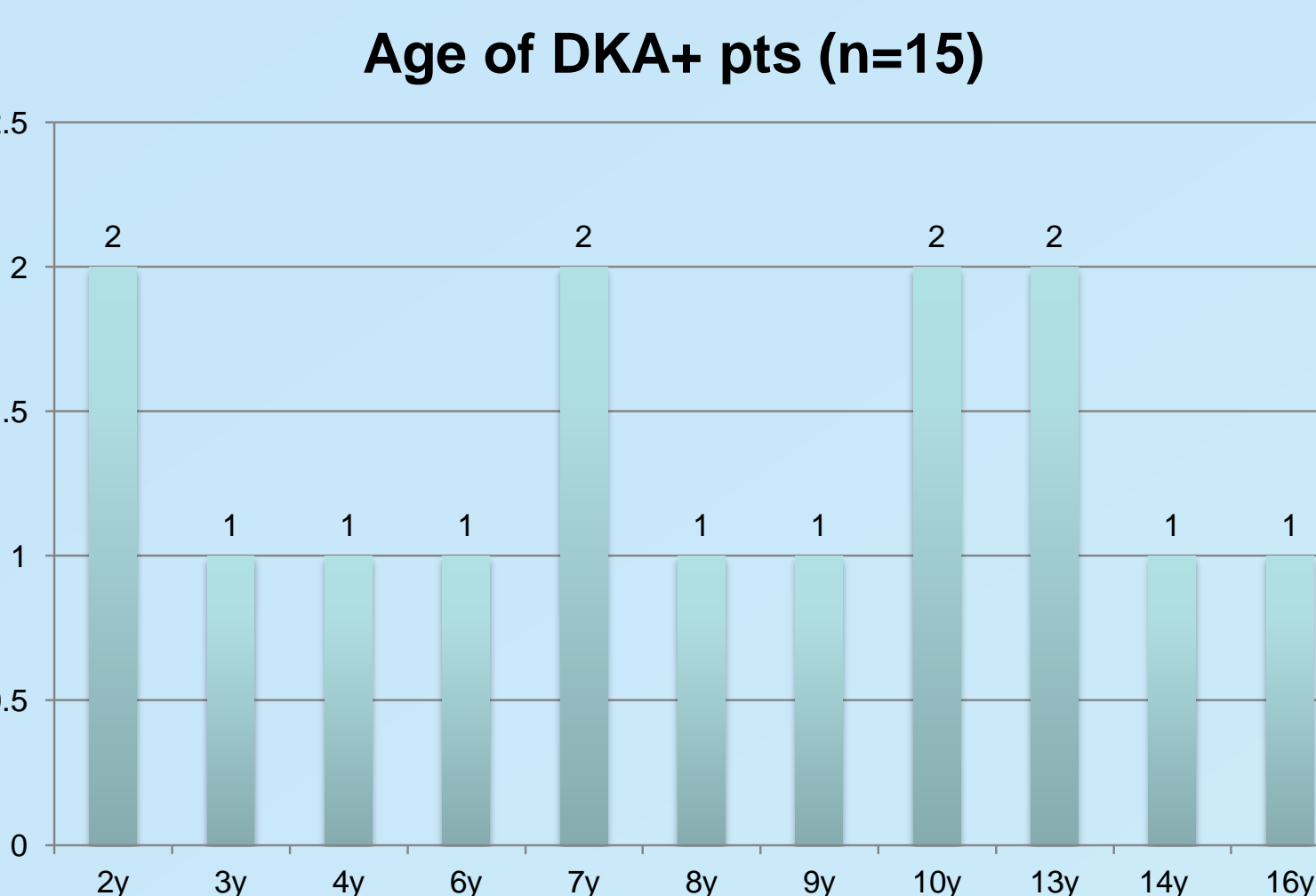

Ethnicity

- $59 \%$ were white British

- $100 \%$ of children with African ethnicity presented in DKA

- compared to $33 \%$ of White British ethnicity $(p=0.0261)$

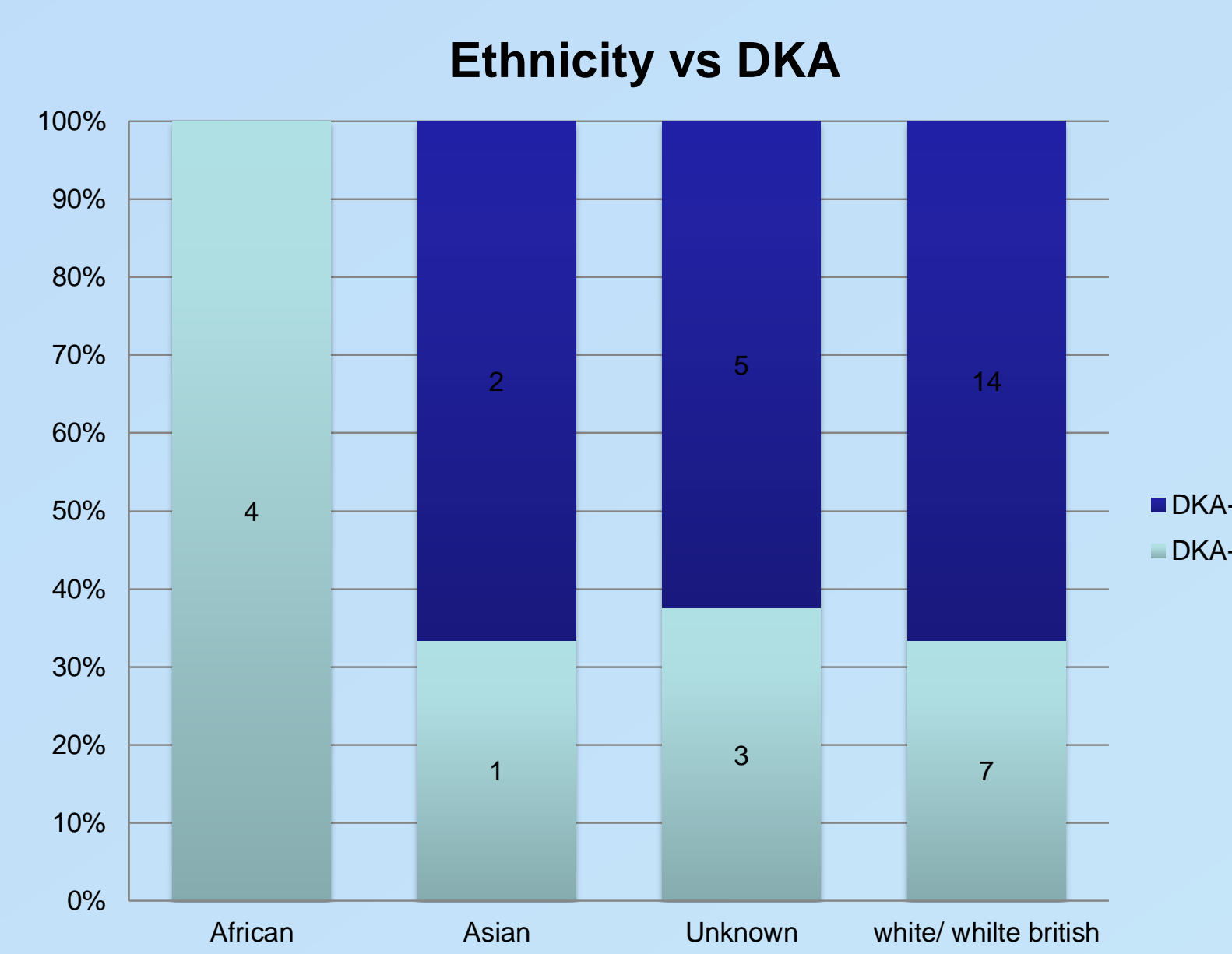

\section{Place of diagnosis}

- More children were diagnosed in hospital rather than by the referring GP

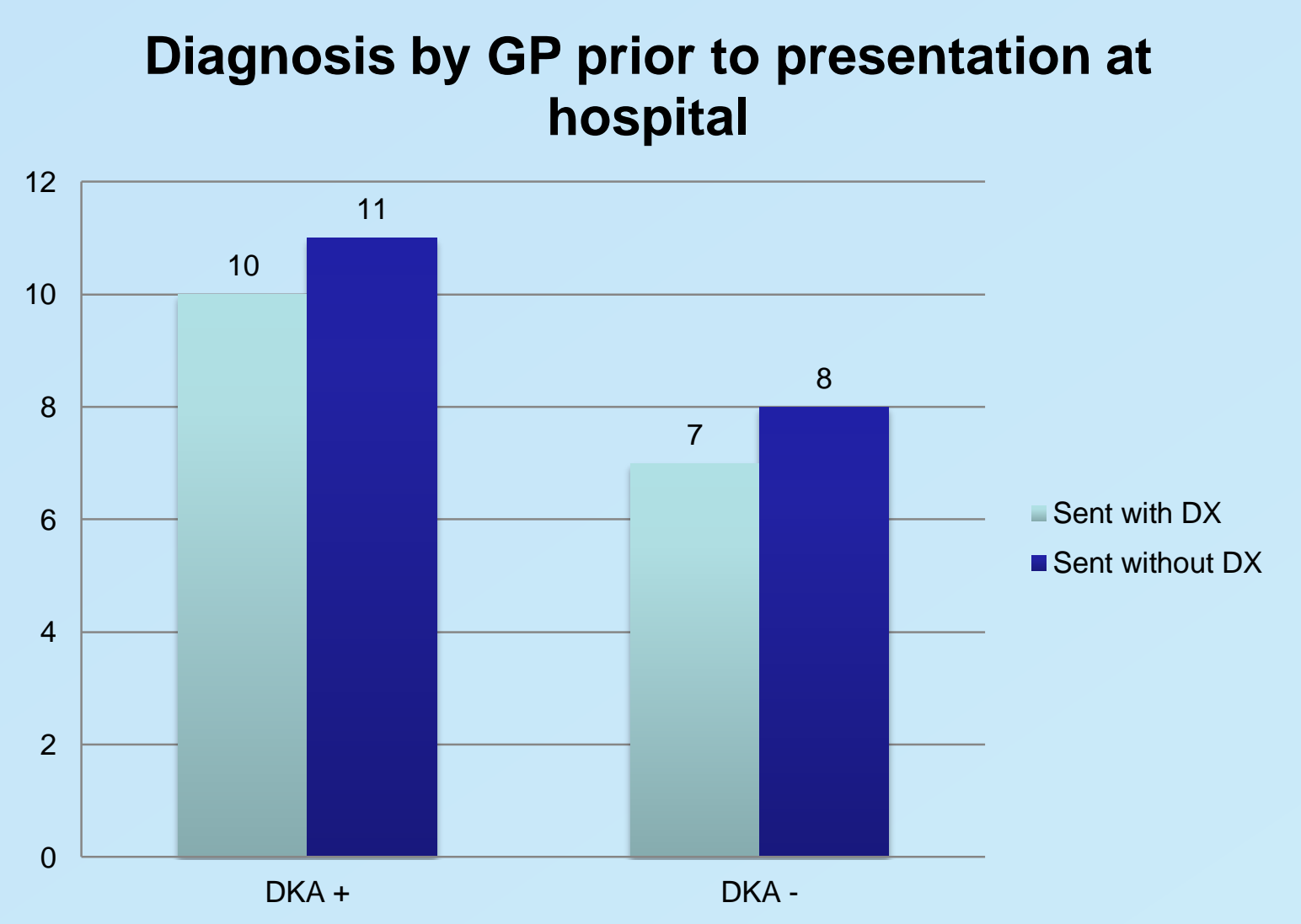

\section{Presentation}

- Commonest symptoms were polydipsia (93\%), polyuria $(86 \%)$, and weight loss (66\%) and were similar in both groups.

- Abdominal pain which was more common in the $\mathrm{DKA}+\operatorname{group}(33 \%$ vs $4.7 \%),(\mathrm{p}=0.059)$

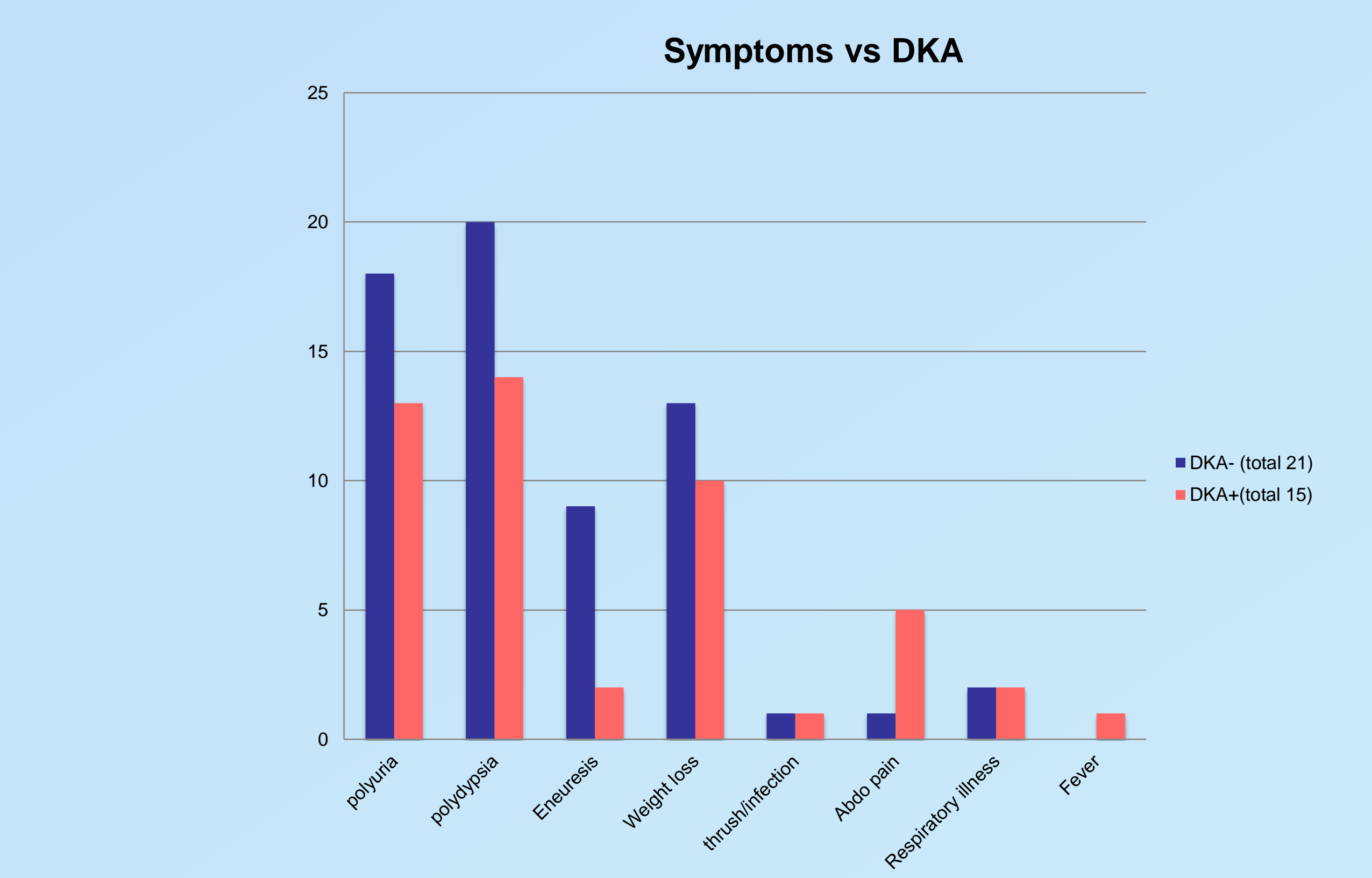

Length of stay

Length of stay was longer in DKA+ (8.8 days; range 5-14) compared to DKA- (6.4 days ; range 4-9) $(p=0.001)$

$\underset{\mathrm{p}=0.001}{\text { Mean Length of } \mathrm{Stay}_{\mathrm{y}} \text { (days) in ward vs DKA }}$

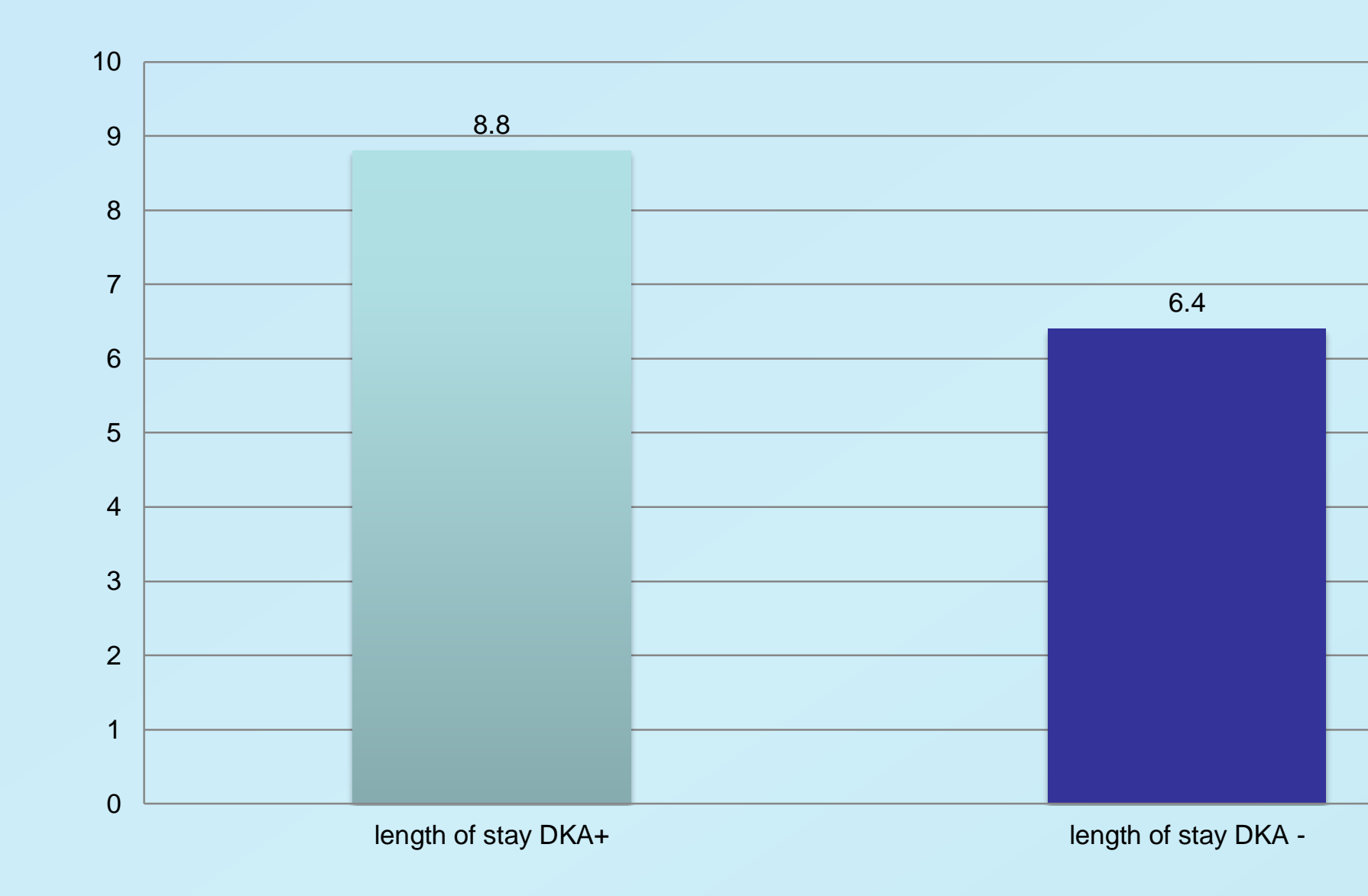

Miscellaneous

There was a family history of Type 1 diabetes in 19\% of children

DKA presentation peaked during winter

There was higher Glucose reading, $\mathrm{HbA1c}$, among DKA+ compared to DKA- Children

\section{CONCLUSIONS}

- Rate of DKA at presentation of T1DM in our patients was higher than that reported in the literature.

- DKA was more common in the Younger age group and African ethnicity

- Cluster of symptoms were similar but abdominal pain was more common in DKA

- Length of stay was significantly longer in patients with DKA

- More patients were diagnosed in hospital than in primary care

- There is a need to increase awareness among the public and primary care health professionals to aid early diagnosis and reduce the burden of DKA. 